Portland State University

PDXScholar

Electrical and Computer Engineering Faculty

Publications and Presentations

8-1-1989

\title{
Spontaneous mode locking in long-cavity xenon lasers
}

Manuela Fe Tarroja

Mohammad Sharafi

Lee W. Casperson

Portland State University

Follow this and additional works at: https://pdxscholar.library.pdx.edu/ece_fac

Part of the Electrical and Computer Engineering Commons

Let us know how access to this document benefits you.

\section{Citation Details}

Manuela Fe H. Tarroja, Mohammad Sharafi, and Lee W. Casperson, "Spontaneous mode locking in longcavity xenon lasers," J. Opt. Soc. Am. B 6, 1564-1573 (1989).

This Article is brought to you for free and open access. It has been accepted for inclusion in Electrical and Computer Engineering Faculty Publications and Presentations by an authorized administrator of PDXScholar. Please contact us if we can make this document more accessible: pdxscholar@pdx.edu. 


\title{
Spontaneous mode locking in long-cavity xenon lasers
}

\author{
Manuela Fe H. Tarroja, ${ }^{*}$ Mohammad Sharafi, and Lee W. Casperson \\ Department of Electrical Engineering, Portland State University, Portland, Oregon 97207
}

Received January 9, 1989; accepted March 10, 1989

\begin{abstract}
Spontaneous mode locking in Fabry-Perot xenon lasers has been experimentally investigated for cavity lengths up to $66 \mathrm{~m}$. Our results show dependence of the number of pulses per round-trip time on the cavity length and the excitation current. Other dynamical effects such as the ringing and modulation of the mode-locked pulses have been observed. A semiclassical model for the self-locking of an inhomogeneously broadened laser is also presented. Numerical results based on this model describe many dynamical features observed in our experiments.
\end{abstract}

\section{INTRODUCTION}

The basic requirement for mode locking is the existence of several modes that are equally spaced in frequency and have constant phase differences. In many mode-locked systems, this phase condition is achieved by the inclusion of either a passive element (e.g., a saturable absorber) or an active element (e.g., a synchronous acousto-optic loss modulator or a synchronously pumped laser amplifier). Early reviews of the different mode-locking mechanisms have been given by Smith $^{1}$ and Allen. ${ }^{2}$ Other mode-locking techniques that have been developed later are based on continuous variation of cavity length ${ }^{3}$ and the rotation of one mirror of a threemirror cavity. ${ }^{4}$ As early as 1965 , however, some laser systems have been observed to exhibit mode locking even in the absence of the above elements or mechanisms. These systems, which include both gas ${ }^{5-27}$ and solid-state ${ }^{28-31}$ lasers, are said to exhibit self-locking or spontaneous mode locking. Although most of the self-locking mechanisms involve only longitudinal modes, simultaneous self-locking of several transverse modes has also been observed. ${ }^{9,14,21}$ It should be noted, however, that the locking that causes longitudinal or transverse pulse formation is different from the cooperative frequency locking in which modes lock to the same optical frequency and form time-independent spatial patterns. ${ }^{32}$

Previous experiments on spontaneous mode locking have been focused on the conditions for its occurrence. Willenbring et al., ${ }^{16,22}$ Nash, ${ }^{11}$ Tanaka et al., ${ }^{4}$ Crowell, ${ }^{5}$ and Statz et $a l .{ }^{10}$ showed that the size of the aperture placed inside the cavity or the $Q$ of the cavity is critical in producing selflocked pulses. The position of the active medium, at least for the Fabry-Perot configuration, also affects the self-locking condition and the type of pulsing, as was shown by Willenbring et al., ${ }^{16}$ Statz et al., ${ }^{10}$ and McClure. ${ }^{6}$ Other parameters that can affect whether the laser is in a locked or an unlocked condition are the excitation current, $3,12,23,26$ the cavity length, $, 6,12,20,22,26,33,34$ and the isotopic content of the laser medium. ${ }^{12,13}$

With the inclusion of a sufficiently small aperture, one can assume that a laser is operating in only one transverse mode, and hence only longitudinal self-locking can occur. In most of tho longitudinal self-locking experiments that have been performed, cavity lengths of only a few meters have been used. When the cavity length is not much longer than the amplifier length, mode-pulling effects due to dispersion in the amplifier would tend to result in unequally spaced mode frequencies. The self-locking condition sometimes observed in such laser systems is usually attributed to the nonlinear interaction between the field and the medium. $1,2,8,9,9,12,22,33-40$

There are several approaches to the theoretical study of self-locking in gas lasers. One approach examines the interactions of the cavity modes that produce beat frequencies (combination tones). ${ }^{33-35}$ Self-locking occurs when a combination tone becomes close to one of the cavity mode frequencies. The condition for self-locking using this approach is formally determined by asserting the time independence of the relative phases of the modes. Another approach is the maximum emission principle, which is based on the idea that the relative phases of the field modes should maximize the rate of stimulated emission. ${ }^{37-40}$ While the above two approaches examine the conditions for self-locking, an alternative method assumes self-locking and examines the response of the medium to the propagating pulses. $8,22,36,41$

Lamb's idea of combination tones has laid the ground for a comprehensive understanding of the self-locking phenomenon. ${ }^{35}$ However, his assumption of slow variation of the field modes compared with the atomic decay time results in the neglect of certain terms in the nonlinear equations coupling the field, polarization, and population difference. The inclusion of these terms has been shown to lead to singlecavity-mode instability, which is an equally essential and interesting nonlinear dynamical effect that involves not only the phases but also the amplitudes of the field modes. Thus it is not surprising that some lasers may undergo transitions between conditions of self-locking, partial locking, or chaotic pulsations. These transitions have been the subject of recent theoretical work on multimode instabilities. ${ }^{42-45}$ Experimentally Halas et al. ${ }^{26}$ have shown the transitions of a $\mathrm{He}-\mathrm{Ne}$ laser from unstable pulsations to locked operation as the excitation current and cavity detuning are varied. Their work as well as that of Weiss et al..$^{24,25}$ and Chetverikov ${ }^{27}$ has shown that low beat frequencies can exhibit transitions from regular pulsations to chaos.

Several reviews have been done on laser instabilities, and some publications devoted to this subject are listed in Refs. 46-48. A thorough investigation of the conditions for multi- 
mode laser instability for the case of a homogeneously broadened two-level system has been done by Risken and Nummedal. ${ }^{41}$ Their approach, which assumes uniformly distributed loss and gain, has been developed further to include other laser configurations. ${ }^{49-52}$ The field numerically generated using models based on the work of Risken and Nummedal shows regular pulsations that have led people to use these models to understand the self-locking phenomena. However, it should be noted that in this model of saturating homogeneously broadened systems only the mode nearest to atomic resonance has net gain, and multimode phenomena such as self-locking appear only with the onset of instability. As in the homogeneous case, self-locking in inhomogeneous systems may also be viewed as a multimode instability. However, inhomogeneously broadened lasers with sufficient cavity length intrinsically have several cavity modes that have enough gain to oscillate simultaneously. The following discussion assumes inhomogeneously broadened lasers since self-locking has been observed mostly in these types of laser.

For a study of self-locking, a relatively long cavity is desirable because in this case mode-pulling effects of the shorter amplifier become negligible. Thus the laser can be expected to stay mode locked for a wider range of experimental conditions. Furthermore, the cavity modes are closely spaced so that the detuning of the cavity modes with respect to the atomic line center can be expected to have little influence on self-locking. A good active medium for long-cavity selflocking experiments is xenon because of its high gain per pass. It permits long-cavity operation, in which mode-pulling effects may be neglected. Although a very-long-cavity xenon laser was first constructed by Linford et al. ${ }^{53}$ the first published report on the xenon self-locking is due to Kim and Marantz, ${ }^{17}$ who used a cavity length of approximately $6 \mathrm{~m}$. The initial investigation of self-locking in a long xenon laser has been done for cavity lengths up to $33 \mathrm{~m} .{ }^{19}$ Apart from that initial work, we are aware of no further studies on the self-locking of a long-cavity laser.

Our experimental investigations focus on a long-cavity laser using the high-gain $3.51-\mu \mathrm{m}$ transition of xenon. For different cavity lengths, the period as well as the features of the self-locked pulsations has been examined as the excitation current is varied. These are presented in Section 2 . In Section 3 a semiclassical model for self-locking based on an inhomogeneously broadened laser is presented and compared with the experimental results.

\section{EXPERIMENTAL RESULTS}

Our experimental setup is shown in Fig. 1. A discharge length equal to $1.1 \mathrm{~m}$ and an i.d. of $0.50 \mathrm{~cm}$ was filled with xenon kept at a constant pressure of approximately $5 \mathrm{~m}$ Torr by a liquid-nitrogen cold trap. The xenon discharge tube was placed close to one of the two flat $99.9 \%$ reflecting mirrors that form the Fabry-Perot cavity. A third flat $99.9 \%$ reflecting mirror was added to fold the beam for long-cavity measurements. To suppress higher transverse modes, an aperture was introduced $2.6 \mathrm{~m}$ away from the discharge tube. A ZnSe beam splitter was used to study the laser beam, which was detected by an InAs detector (with a rise time of 7 nsec). To obtain good amplification, the signal was first amplified by a Hewlett-Packard dual amplifier (Model 8447D) whose output was fed into the Tektronix amplifier

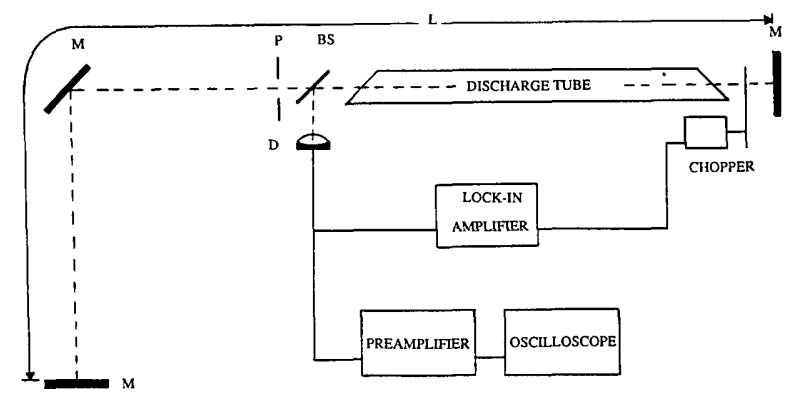

Fig. 1. Experimental setup: M's, mirrors; BS, beam splitter; D, InAs detector; $\mathrm{P}$, pinhole; $L$, cavity length. The tube is filled with 5 $m$ Torr of xenon. The path of the beam is represented by the dashed line.

(a)

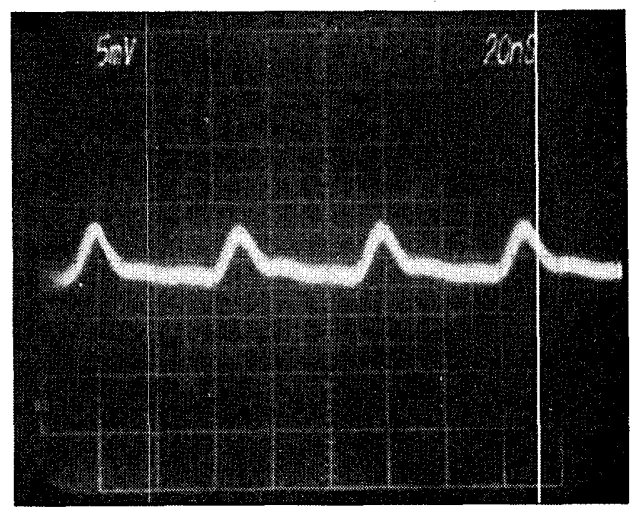

(b)

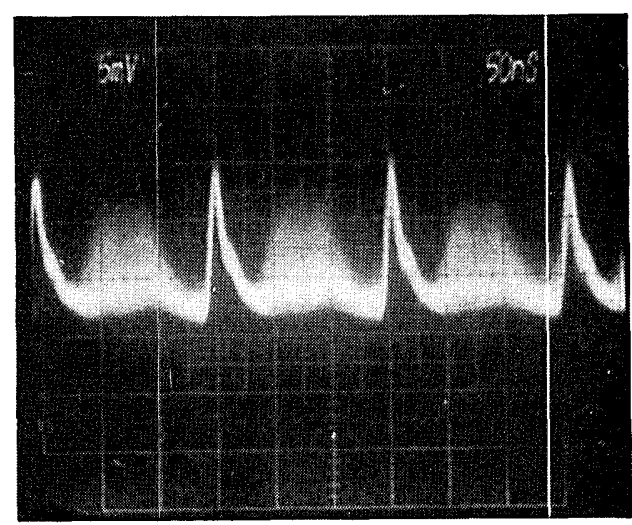

(c)

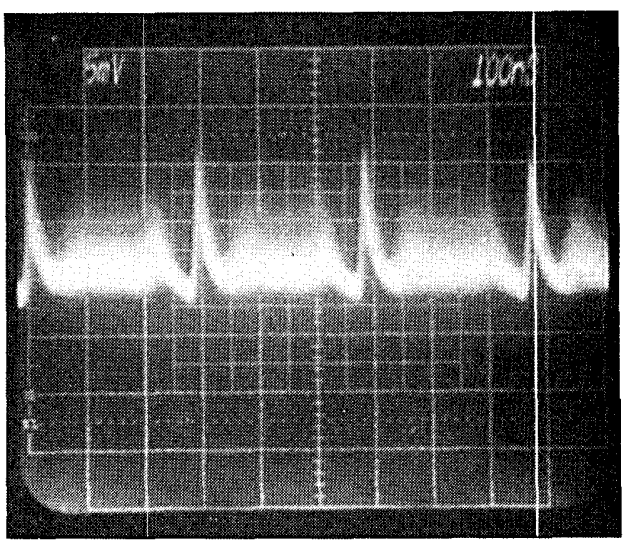

Fig. 2. Temporal properties of the self-locked laser for (a) $L=7.4$ $\mathrm{m}, r=1.8$; (b) $L=22.8 \mathrm{~m}, r=1.6$; and (c) $L=44.2, r=1.6$. The round-trip times are 49, 152, and $295 \mathrm{nsec}$ for (a), (b), and (c), respectively. 
(a)

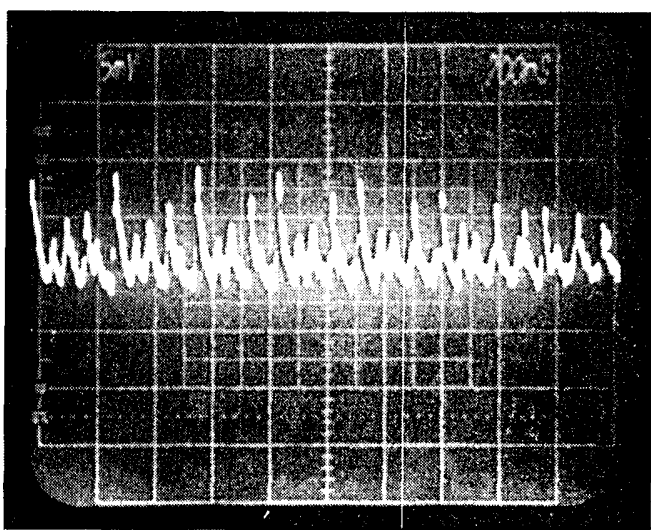

(b)

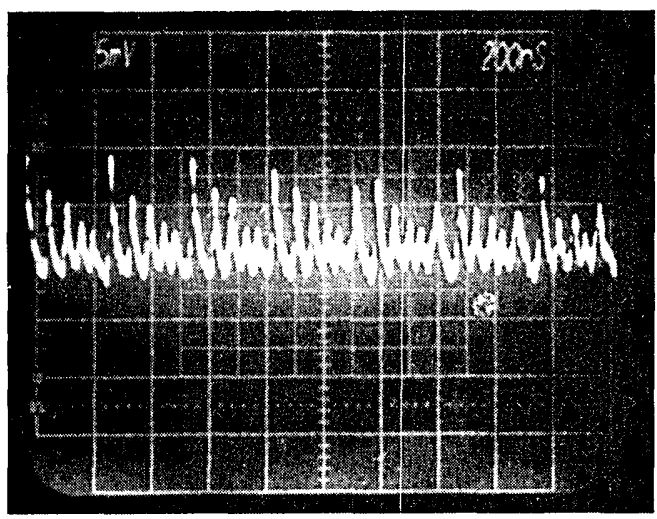

(c)

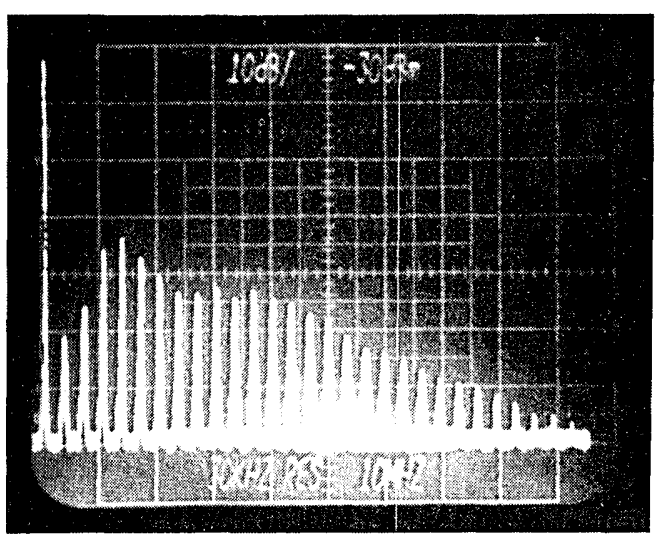

Fig. 3. Temporal and spectral properties of the self-locked laser for $L=44.2 \mathrm{~m}$ and $r=1.6$. For a round-trip time of $295 \mathrm{nsec}$, three, four, and five pulses are observed as seen in the single-trace displays, (a) and (b), of the signals. The corresponding spectrum is shown in (c). The temporal properties are shown in single-trace oscilloscope displays.

plug-in (Model 7A26). The storage oscilloscope (Tektronix Model 7834) displayed either the temporal characteristic of the signal or its spectrum, which is generated by a $7 \mathrm{~L} 12$ Tektronix spectrum analyzer. For average power measurements, the signal was chopped at a frequency of $85 \mathrm{~Hz}$ and measured with a lock-in amplifier. The spontaneous mode locking was studied for different cavity lengths up to $66 \mathrm{~m}$ as the excitation current and aperture diameter were varied.

Our results show that the number of pulses per round-trip time increases with increasing cavity length. Figure 2 includes multitrace displays of the temporal properties of selfpulsing for different cavity lengths. The fuzziness of the pulses associated with the longer cavities is due to the modu- lation of the pulses that causes the laser to oscillate with one number lower or higher than the dominant number of pulses observed. Figures 3(a) and 3(b) show this type of modulation for $L=44.2 \mathrm{~m}$, which corresponds to a round-trip time of $294 \mathrm{nsec}$. The single-trace display shows three, four, and five pulses per round-trip time. This is also evident in the corresponding spectrum in Fig. 3(c), which shows strong third, fourth, and fifth frequency peaks. A typical plot of the pulse modulation for a longer temporal range is shown in Fig. 4.

The observed number of pulses is also limited by the value of $r$ permitted by the experimental setup. For the planeparallel resonator configuration used in our experiments, diffraction losses become very large for the longer cavity lengths. As a result the threshold current also increases with length, and the maximum values of $r$ attainable for longer cavities are smaller. In spite of this experimental limitation, it is clear from Table 1 that the maximum observed number of pulses increases with increasing cavity length.

For a fixed cavity length the number of pulses per roundtrip time increases with increasing excitation. Figures 5 and 6 show the temporal behavior of the self-locked pulses for cavity lengths equal to 22.5 and $15 \mathrm{~m}$, respectively, as a function of the excitation parameter, $r$ (which is equal to the ratio of the excitation current to its value at threshold). Figure 5 shows that the system evolves from a stable two pulses per round-trip time of $150 \mathrm{nsec}$ close to threshold to three pulses per round-trip time at $r=2.1$. This evolution from two to three pulses per round-trip time is manifested in the spectral characteristics of the pulses as shown in Fig. 7. Strong second and third frequency peaks correspond to two

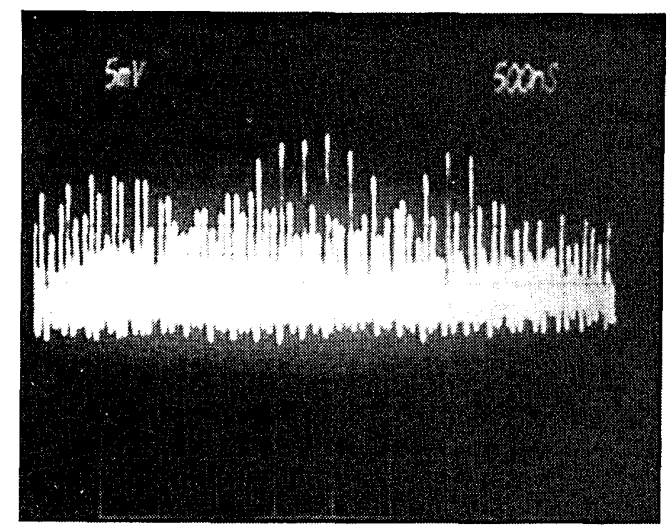

Fig. 4. Modulation of the self-locked pulses for $L=30 \mathrm{~m}$ and $r=$ 2.0. The round-trip time is equal to $200 \mathrm{nsec}$. The signal shown is a single-trace oscilloscope display.

Table 1. Maximum Number of Pulses for Various Cavity Lengths

\begin{tabular}{ccc}
\hline $\begin{array}{c}\text { Cavity Length } \\
(\mathrm{m})\end{array}$ & $r_{\max }$ & Number of Pulses \\
\hline 7.4 & 2.8 & 1 \\
15.0 & 2.3 & 2 \\
22.5 & 2.1 & 3 \\
30.0 & 2.0 & 4 \\
44.2 & 1.6 & 5 \\
66.0 & 1.3 & 6 \\
\hline
\end{tabular}



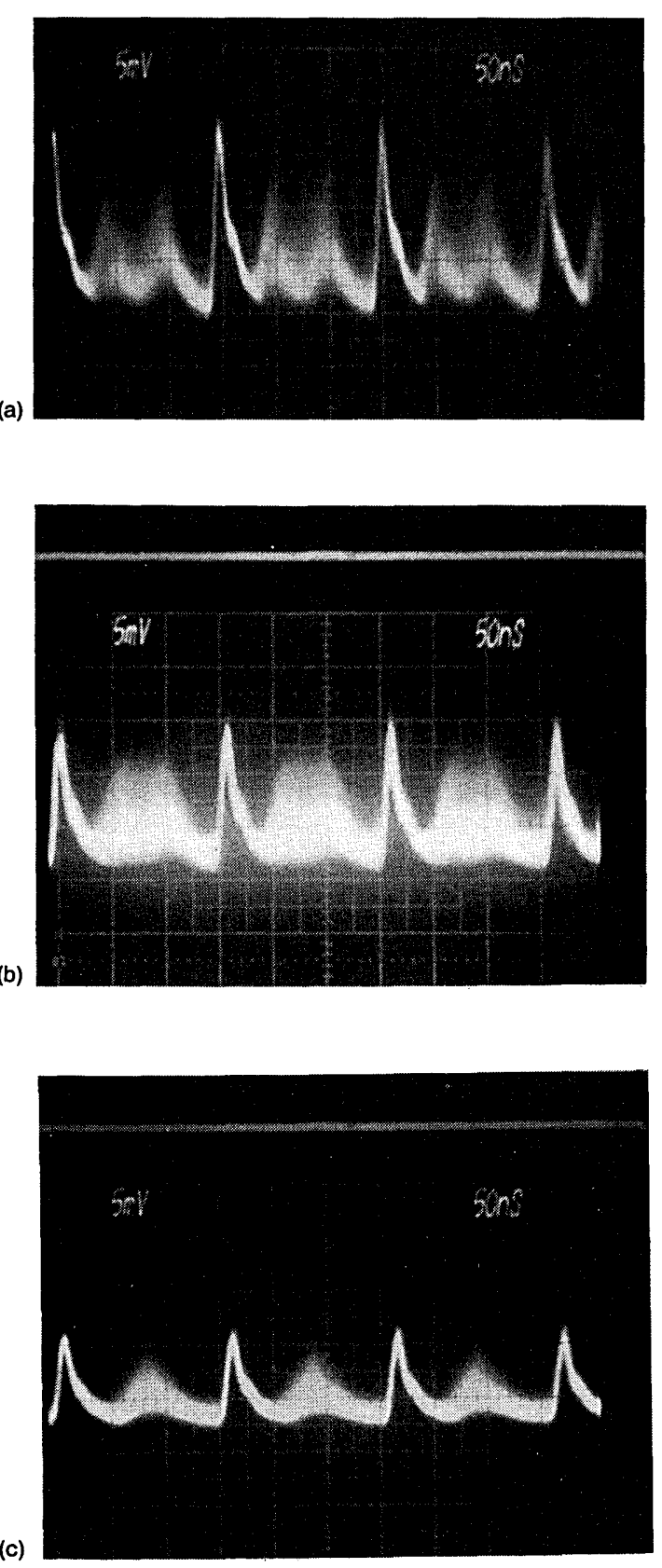

(c)

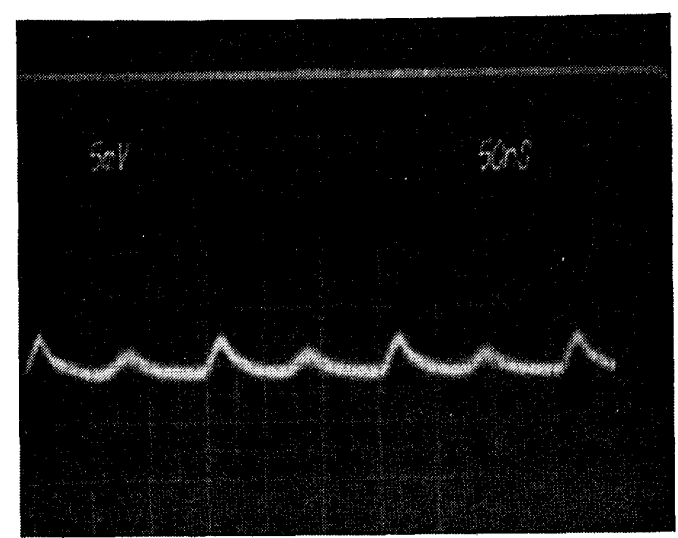

Fig. 5. Temporal properties of the self-locked laser for $L=22.5 \mathrm{~m}$ and for $r$ equal to (a) 2.1 ; (b) 2.0 ; (c) 1.5 ; and (d) 1.2 . and three pulses, respectively, per round-trip time. Figure 6 , on the other hand, shows the evolution from one pulse to two pulses per round-trip time of 100 nsec. In both cases the transition as well as the final state is characterized by the fuzziness between the strong $2 L / c$ pulses (where $c$ is the velocity of light and $L$ is the cavity length), which has been associated previously with the modulation of the individual pulses. As suggested by Fig. 7, which shows the narrowing of the spectral peaks and the disappearance of the lowfrequency components for sufficiently low excitation, the pulse modulation decreases with decreasing excitation current. Just above threshold, the pulses are periodic.

Increasing excitation also decreases the width of the fundamental pulse. Our experimental measurements confirm the results summarized in Fig. 8, which shows a plot of the
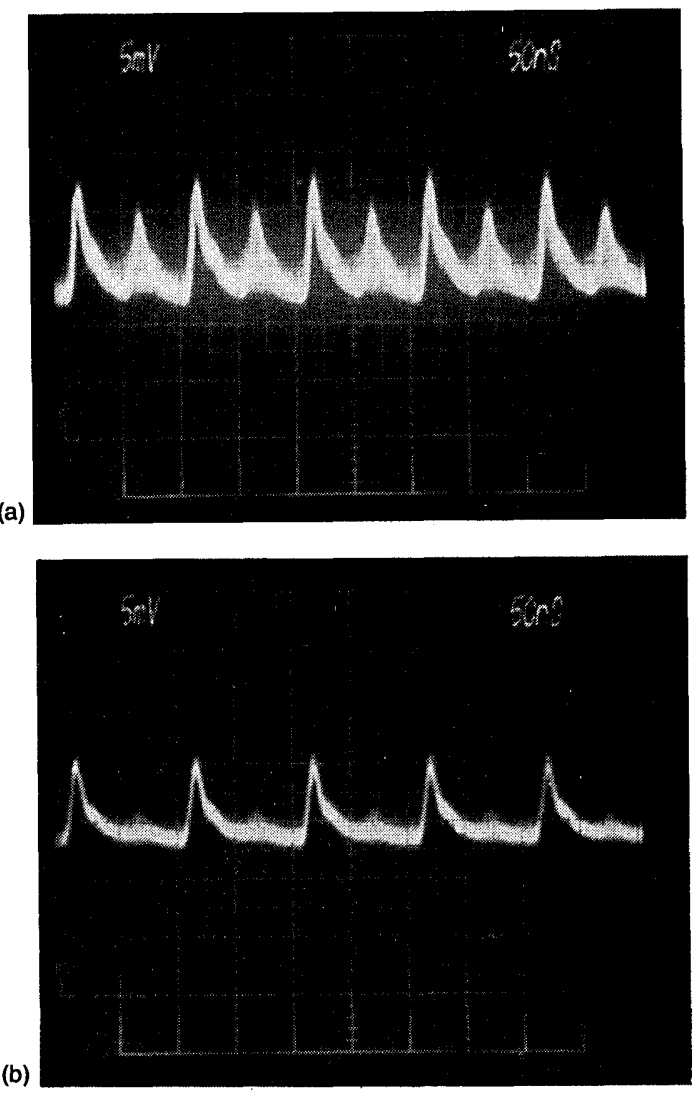

(b)

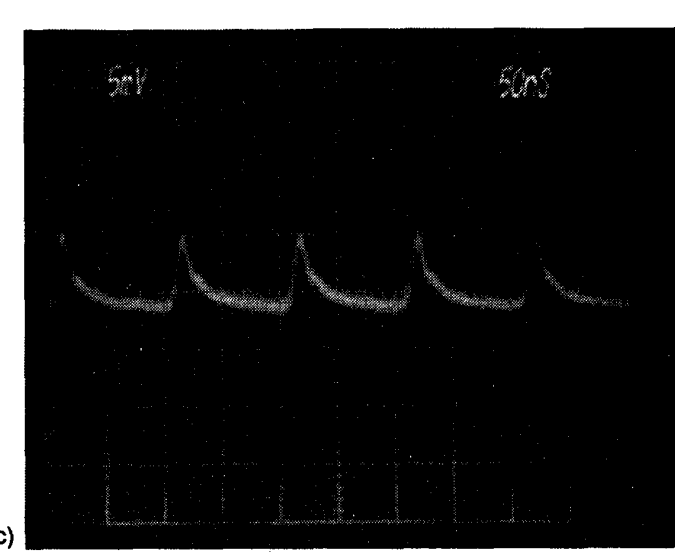

Fig. 6. Temporal properties of the self-locked laser for $L=15 \mathrm{~m}$ and for $r$ equal to (a) 2.3 ; (b) 2.0 ; and (c) 1.6 . 
(a)

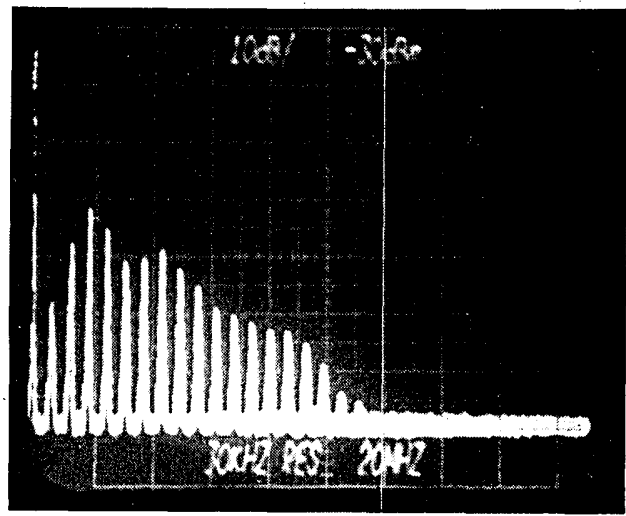

(b)

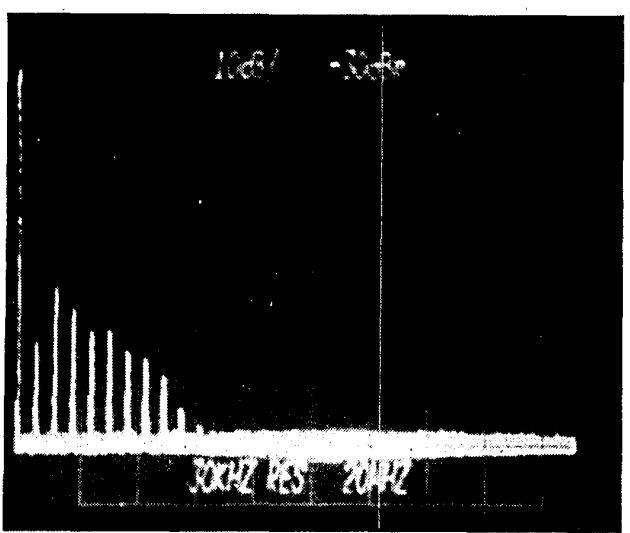

Fig. 7. Spectral properties of the self-locked laser for $L=22.5 \mathrm{~m}$ and for $r$ equal to (a) 2.1 and (b) 1.2 .

width as a function $\left(g-g_{\text {th }}\right)^{-1 / 2}$, where $g$ is the gain and $g_{\text {th }}$ is its value at threshold. This is consistent with the prediction of a decrease of the pulse width as a result of an increase in the number of modes that reach threshold when the gain is increased. ${ }^{19}$

Another characteristic feature of the self-locked pulses that is evident in the temporal plots (Figs. 2, 3, 5, and 6) shown so far is the ringing feature of the fundamental pulse. This is clearly evident in Fig. 2(a). For longer cavity lengths, the asymmetry of the fundamental pulse suggests the ringing, which may be too fast to be resolved fully by our detector.

The excitation scan of long-cavity lasers is limited by their high threshold. In contrast, short cavity lengths have lower excitation current thresholds, which allow the examination of the self-pulsing characteristics corresponding to large $r$. The evolution of the spectra as a function of $r$ for a cavity length equal to $4.6 \mathrm{~m}$ is shown in Fig. 9. The figure shows an increase in the width of the frequency peaks as $r$ is increased. Furthermore, more complex spectral profiles with low-frequency components are observed for higher excitation current. The presence of low-frequency components has been associated with unequal frequency spacings among cavity modes. Thus this suggests that the laser is only partially self-locked if at all. A similar trend in the spectral properties of the signal is observed as the aperture introduced in the cavity is varied. The above results for the short cavity length are consistent with previous experimental results that suggest that a judicious choice of the aperture size, the $Q$ of the cavity, or the degree of excitation may be required if self-locking is to be observed.

Self-locking, at least for the xenon Fabry-Perot configura- tions used here, is manifested in trains of periodic pulses with frequencies that are integral multiples of $c / 2 L$. This is evident for all cavity lengths. The results for the shorter cavity lengths (e.g., $4.6 \mathrm{~m}$ ) sometimes also show sharp lowfrequency components, which may be attributed to the small differences among the cavity frequencies. Although the existence of these beat frequencies is consistent with Lamb's approach using the concept of combination tones, the evolution of these low-frequency components to a continuous spread of low beat frequencies, and the broadening of the frequency peaks as the excitation and the cavity length tuning are varied, are more suggestive of the instabilities of the individual cavity modes. The association of the low frequencies with the laser instability is consistent with the results found by Halas et al. ${ }^{26}$ and Weiss et al. ${ }^{24,25}$ This laser instability has been ignored in Lamb's theory for multiple-mode operation of the laser but has been separately studied by others in laser systems that permit only one cavity mode. Initial experimental and theoretical investigations of this single-mode instability were done by Casperson. ${ }^{19,54}$

The above results show that the laser's tendencies to exhibit single-cavity-mode instability and to self-lock can be exhibited simultaneously. These tendencies can be attributed to two equally significant nonlinear mechanisms-one that tends to force the phases of the cavity modes to be constant in time, thus producing self-locking, and the other that tends to disrupt equal cavity frequency spacings owing to the nonlinear coupling among the field, polarization, and population difference of each mode. Thus, in addition to mode pulling effects, single-cavity-mode instabilities, which have been shown to depend on both the excitation current and detuning, may also be responsible for the critical dependence of self-locking on the experimental conditions, particularly for short cavity lengths. It is therefore not surprising that self-locking in earlier experiments has been observed only with a judicious choice of experimental parameters.

\section{THEORETICAL RESULTS}

Self-locking of long-cavity lasers using the multimode approach has not yet been thoroughly explored because of the complicated theoretical and numerical calculations involving many modes. For a sufficiently long cavity for which the

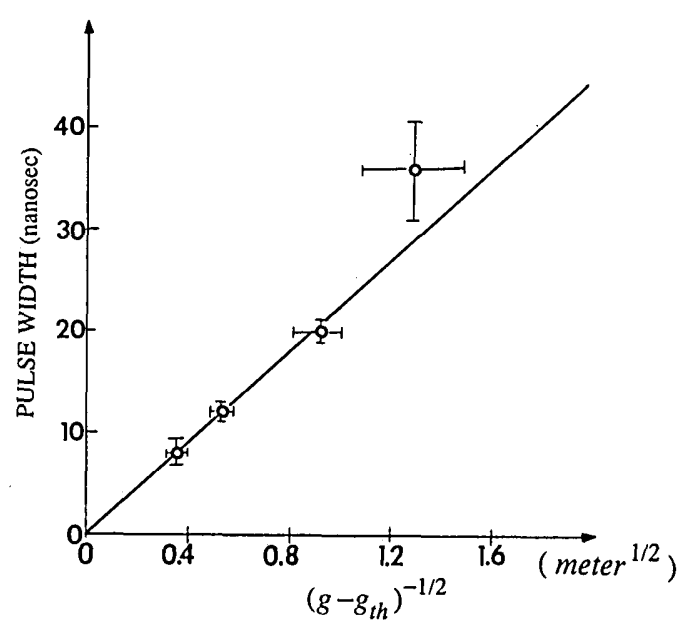

Fig. 8. Pulse width versus $\left(g-g_{\text {th }}\right)^{-1 / 2}$, where $g_{\text {th }}$ is the gain at threshold. Taken from Ref. 19. 
(a)

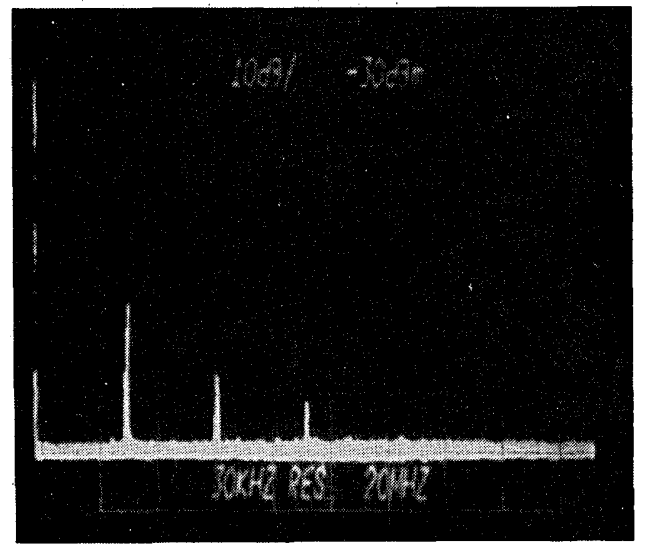

(b)

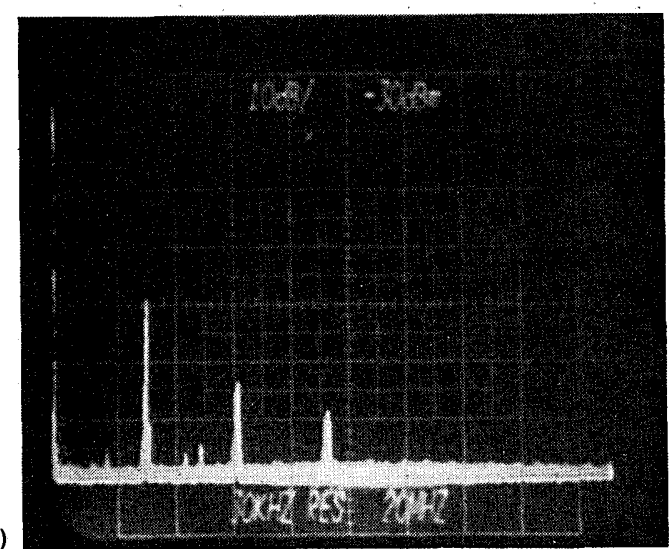

(c)

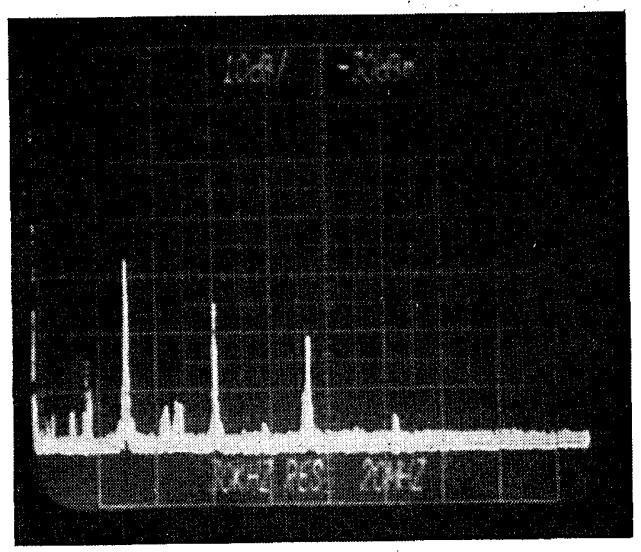

(d)

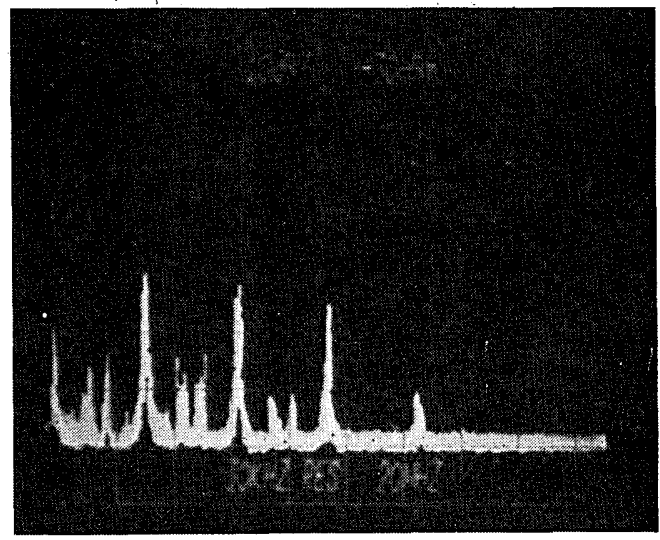

Fig. 9. Spectral properties of the self-locked laser for $L=4.6 \mathrm{~m}$ and for $r$ equal to (a) 1.8 ; (b) 2.2 ; (c) 2.7 ; (d) 3.4 .

multimode approach becomes theoretically and numerically cumbersome, the laser may be treated as an amplifying medium that responds to a train of mode-locked pulses. Smith used this approach for inhomogeneously broadened systems but used rate equations, ${ }^{8}$ while Risken and Nummedal used a semiclassical model for homogeneously broadened twolevel systems. ${ }^{41}$ A similar idea of a laser as an amplifier has been utilized in our model, which is presented below. The semiclassical approach is assumed because it has the advantage of rigorously incorporating the nonlinear effects that arise from the coupling among the field, population difference, and polarization, which have been approximated in the previous work on self-locking. ${ }^{1-3,8-10,22,23,33-40}$ Because many self-locking laser systems, including the one described in Section 2, are inhomogeneously broadened, this type of broadening is also assumed here.

The starting point of our semiclassical model for selflocking is a set of Maxwell-Schrödinger equations that have been used to study laser instabilities. ${ }^{55}$ Inhomogeneous broadening due to the Doppler effect and a distribution of intrinsic resonance frequencies are assumed, and the effects of velocity-changing collisions are incorporated. The density matrix equations are

$$
\begin{aligned}
\left(\frac{\partial}{\partial t}+v \frac{\partial}{\partial z}\right) \rho_{a b}\left(v, \omega_{\alpha}, z, t\right) & \\
= & -\left(i \omega_{\alpha}+\gamma\right) \rho_{a b}\left(v, \omega_{\alpha}, z, t\right) \\
& \quad-\frac{i \mu}{\hbar} E(z, t)\left[\rho_{a a}\left(v, \omega_{\alpha}, z, t\right)-\rho_{b b}\left(v, \omega_{\alpha}, z, t\right)\right]
\end{aligned}
$$

$$
\begin{aligned}
&\left(\frac{\partial}{\partial t}+v \frac{\partial}{\partial z}\right) \rho_{a a}\left(v, \omega_{\alpha}, z, t\right) \\
&= \lambda_{a}\left(v, \omega_{\alpha}, z, t\right)-\gamma_{a} \rho_{a a}\left(v, \omega_{\alpha}, z, t\right) \\
&+\left[\frac{i \mu}{\hbar} E(z, t) \rho_{b a}\left(v, \omega_{\alpha}, z, t\right)+c . c .\right] \\
& \quad-\int_{-\infty}^{\infty} \Gamma_{a}\left(v^{\prime}, v\right) \rho_{a a}\left(v, \omega_{\alpha}, z, t\right) \mathrm{d} v^{\prime} \\
&+\int_{-\infty}^{\infty} \Gamma_{a}\left(v, v^{\prime}\right) \rho_{a a}\left(v^{\prime}, \omega_{\alpha}, z, t\right) \mathrm{d} v^{\prime} \\
&=\lambda_{b}\left(v, \omega_{\alpha}, z, t\right)-\gamma_{b} \rho_{b b}\left(v, \omega_{\alpha}, z, t\right) \\
&+\gamma_{a b} \rho_{a a}\left(v, \omega_{\alpha}, z, t\right)-\left[\frac{\partial \mu}{\hbar} E(z, t) \rho_{b a}\left(v, \omega_{\alpha}, z, t\right)+c . c .\right. \\
& \quad-\int_{-\infty}^{\infty} \Gamma_{b}\left(v^{\prime}, v\right) \rho_{b b}\left(v, \omega_{\alpha}, z, t\right) \mathrm{d} v^{\prime} \\
&+\int_{-\infty}^{\infty} \Gamma_{b}\left(v, v^{\prime}\right) \rho_{b b}\left(v^{\prime}, \omega_{\alpha}, z, t\right) \mathrm{d} v^{\prime} \\
& \rho_{b a}\left(v, \omega_{\alpha}, z, t\right)=\rho_{a b} *\left(v, \omega_{\alpha}, z, t\right)
\end{aligned}
$$

where $v$ is the atomic velocity and $\omega_{\alpha}$ is the center frequency of the laser transition for members of the atomic class $\alpha$. $\gamma_{a}$ and $\gamma_{b}$ are the total decay rates of levels $a$ and $b$, respectively, while $\gamma_{a b}$ is the decay rate from level a to level $b$. The total 
decay rate of the off-diagonal elements is $g$, which is equal to $\left(\gamma_{\mathrm{a}}+\gamma_{\mathrm{b}}\right) / 2+\gamma_{\mathrm{ph}}$, where $\gamma_{\mathrm{ph}}$ is the rate of phase-interrupting collisions. $\lambda_{a}$ and $\lambda_{b}$ are the pumping rates of level $a$ and level $b$, respectively. The integral terms in the equations correspond to the spectral cross relaxation. $\Gamma_{a}\left(v^{\prime}, v\right) \mathrm{d} v^{\prime}$ represents the rate at which atoms in level a having velocity $v$ will be bumped to a velocity range between $v^{\prime}$ and $v^{\prime}+\mathrm{d} v^{\prime}$.

The electric-field equation is described by Maxwell's wave equation for a linearly polarized wave traveling in the laser medium as shown below:

$$
\frac{\partial^{2} E(z, t)}{\partial z^{2}}-\mu_{0} \sigma \frac{\partial E(z, t)}{\partial t}-\mu_{0} \epsilon_{0} \frac{\partial^{2} E(z, t)}{\partial t^{2}}=\mu_{0} \frac{\partial^{2} P(z, t)}{\partial t^{2}} \text {. }
$$

The macroscopic polarization $P$ is related to the off-diagonal elements of the density matrix by

$$
P(z, t)=\int_{0}^{\infty} \int_{-\infty}^{\infty} \mu \rho_{a b}\left(v, \omega_{\alpha}, z, t\right) \mathrm{d} v \mathrm{~d} \omega_{\alpha}+\text { c.c. }
$$

Assuming a ring-cavity configuration, the field and polarization can be represented by traveling waves, where slowly varying amplitudes, $E^{\prime}(z, t)$ and $P^{\prime}\left(v, \omega_{\alpha}, z, t\right)$, of the field and polarization, respectively, may be factored out as follows:

$$
\begin{gathered}
E(z, t)=1 / 2 E^{\prime}(z, t) \exp (i k z-i \omega t)+\text { c.c. } \\
\rho_{a b}\left(v, \omega_{\alpha}, z, t\right)=P^{\prime}\left(v, \omega_{\alpha}, z, t\right) \exp (i k z-i \omega t) / 2 \mu .
\end{gathered}
$$

By following the same procedure as in Ref. 55 and using the slowly varying amplitude and the rotating wave approximations, Eqs. (1)-(3) and (5) may be written as

$$
\begin{aligned}
& \frac{\partial P_{r}\left(v, \omega_{\alpha}, z, t\right)}{\partial t}+v \frac{\partial P_{r}\left(v, \omega_{\alpha}, z, t\right)}{\partial z} \\
&=-\left(\omega-\omega_{\alpha}-k v\right) P_{i}\left(v, \omega_{\alpha}, z, t\right)-\gamma P_{r}\left(v, \omega_{\alpha}, z, t\right) \\
&+\frac{\mu^{2}}{\hbar} E_{i}\left(v, \omega_{\alpha}, z, t\right) D(z, t) .
\end{aligned}
$$

$$
\begin{array}{r}
\frac{\partial P_{i}\left(v, \omega_{\alpha}, z, t\right)}{\partial t}+v \frac{\partial P_{i}\left(v, \omega_{\alpha}, z, t\right)}{\partial z} \\
=\left(\omega-\omega_{\alpha}-k v\right) P_{r}\left(v, \omega_{\alpha}, z, t\right)-\gamma P_{i}\left(v, \omega_{\alpha}, z, t\right) \\
\quad-\frac{\mu^{2}}{\hbar} E_{r}\left(v, \omega_{\alpha}, z, t\right) D(z, t)
\end{array}
$$

$$
\begin{aligned}
\frac{\partial M\left(v, \omega_{\alpha}, z, t\right)}{\partial t}+v \frac{\partial M\left(v, \omega_{\alpha}, z, t\right)}{\partial z} \\
=\lambda_{a}+\lambda_{b}-\frac{\gamma_{a}-\gamma_{a b}-\gamma_{b}}{2} \\
\quad \times D\left(v, \omega_{\alpha}, z, t\right)-\frac{\gamma_{a}-\gamma_{a b}+\gamma_{b}}{2} M\left(v, \omega_{\alpha}, z, t\right) \\
\quad-\frac{1}{2} \int_{-\infty}^{\infty} \Gamma_{a}\left(v^{\prime}, v\right)\left[M\left(v, \omega_{\alpha}, z, t\right)+D\left(v, \omega_{\alpha}, z, t\right)\right] \mathrm{d} v^{\prime} \\
+\frac{1}{2} \int_{-\infty}^{\infty} \Gamma_{a}\left(v, v^{\prime}\right)\left[M\left(v^{\prime}, \omega_{\alpha}, z, t\right)+D\left(v^{\prime}, \omega_{\alpha}, z, t\right)\right] \mathrm{d} v^{\prime} \\
-\frac{1}{2} \int_{-\infty}^{\infty} \Gamma_{b}\left(v^{\prime}, v\right)\left[M\left(v, \omega_{\alpha}, z, t\right)-D\left(v, \omega_{\alpha}, z, t\right)\right] \mathrm{d} v^{\prime} \\
+\frac{1}{2} \int_{-\infty}^{\infty} \Gamma_{b}\left(v, v^{\prime}\right)\left[M\left(v^{\prime}, \omega_{\alpha}, z, t\right)-D\left(v^{\prime}, \omega_{\alpha}, z, t\right)\right] \mathrm{d} v^{\prime},
\end{aligned}
$$

$$
\begin{aligned}
& \frac{\partial D\left(v, \omega_{\alpha}, z, t\right)}{\partial t}+v \frac{\partial D\left(v, \omega_{\alpha}, z, t\right)}{\partial z} \\
& =\lambda_{a}-\lambda_{b}-\frac{\gamma_{a}+\gamma_{a b}+\gamma_{b}}{2} D\left(v, \omega_{\alpha}, z, t\right) \\
& -\frac{\gamma_{a}+\gamma_{a b}-\gamma_{b}}{2} M\left(v, \omega_{\alpha}, z, t\right) \\
& +\frac{i}{\hbar}\left[E_{r}(z, t) P_{i}\left(v, \omega_{\alpha}, z, t\right)-E_{i}(z, t) P_{r}\left(v, \omega_{\alpha}, z, t\right)\right] \\
& -\frac{1}{2} \int_{-\infty}^{\infty} \Gamma_{a}\left(v^{\prime}, v\right)\left[M\left(v, \omega_{\alpha}, z, t\right)+D\left(v, \omega_{\alpha}, z, t\right)\right] \mathrm{d} v^{\prime} \\
& +\frac{1}{2} \int_{-\infty}^{\infty} \Gamma_{a}\left(v, v^{\prime}\right)\left[M\left(v^{\prime}, \omega_{\alpha}, z, t\right)+D\left(v^{\prime}, \omega_{\alpha}, z, t\right)\right] \mathrm{d} v^{\prime} \\
& +\frac{1}{2} \int_{-\infty}^{\infty} \Gamma_{b}\left(v^{\prime}, v\right)\left[M\left(v, \omega_{\alpha}, z, t\right)-D\left(v, \omega_{\alpha}, z, t\right)\right] \mathrm{d} v^{\prime} \\
& -\frac{1}{2} \int_{-\infty}^{\infty} \Gamma_{b}\left(v, v^{\prime}\right)\left[M\left(v^{\prime}, \omega_{\alpha}, z, t\right)-D\left(v^{\prime}, \omega_{\alpha}, z, t\right)\right] \mathrm{d} v^{\prime}, \\
& \frac{\partial E_{r}(z, t)}{\partial t}+v_{f} \frac{\partial E_{r}(z, t)}{\partial z}=-\frac{(\omega-\Omega)}{2} E_{i}(z, t)-\frac{1}{2 t_{c}} E_{r}(z, t) \\
& -\frac{\omega}{2 \epsilon_{0}} \int_{0}^{\infty} \int_{-\infty}^{\infty} P_{i}\left(v, \omega_{\alpha}, z, t\right) \mathrm{d} v \mathrm{~d} \omega_{\alpha}, \\
& \frac{\partial E_{i}(z, t)}{\partial t}+v_{f} \frac{\partial E_{i}(z, t)}{\partial z}=+\frac{(\omega-\Omega)}{2} E_{r}(z, t)-\frac{1}{2 t_{c}} E_{i}(z, t) \\
& +\frac{\omega}{2 \epsilon_{0}} \int_{0}^{\infty} \int_{-\infty}^{\infty} P_{r}\left(v, \omega_{\alpha}, z, t\right) \mathrm{d} v \mathrm{~d} \omega_{\alpha} .
\end{aligned}
$$

In the above equations $P^{\prime}$ and $E^{\prime}$ are expressed in terms of their real and imaginary parts and the density-matrix components are replaced by the new variables $M=\rho_{a a}+\rho_{b b}$ and $D=\rho_{a a}-\rho_{b b} . \quad v_{f}$ is the phase velocity of the field equal to $k /$ $\left(\epsilon_{0} \mu_{0} \omega\right)$. The spectral cross-relaxation kernels of strong collisions are assumed since the primary consequence of the weak velocity-changing collisions is to broaden the effective homogeneous linewidth. For strong velocity-changing collisions, $\Gamma_{a}$ and $\Gamma_{b}$ are given below:

$$
\begin{aligned}
& \Gamma_{a}\left(v, v^{\prime}\right)=\frac{\Gamma_{a}}{u \pi^{1 / 2}} \exp \left(-\frac{v^{2}}{u^{2}}\right), \\
& \Gamma_{b}\left(v, v^{\prime}\right)=\frac{\Gamma_{b}}{u \pi^{1 / 2}} \exp \left(-\frac{v^{2}}{u^{2}}\right),
\end{aligned}
$$

where $u$ is the most probable velocity of the atoms.

As in Ref. 55, the field and polarization amplitudes, the sum and difference of the density matrix elements, and the pump rates are normalized as follows:

$$
\begin{aligned}
A_{r}(z, t)= & \left(\frac{\gamma_{a}{ }^{\prime}-\gamma_{a b}+\gamma_{b}{ }^{\prime}}{2 \gamma \gamma_{a}{ }^{\prime} \gamma_{b}{ }^{\prime}}\right)^{1 / 2} \frac{\mu E_{r}(z, t)}{\hbar} \\
A_{i}(z, t)= & \left(\frac{\gamma_{a}{ }^{\prime}-\gamma_{a b}+\gamma_{b}{ }^{\prime}}{2 \gamma \gamma_{a}^{\prime} \gamma_{b}^{\prime}}\right)^{1 / 2} \frac{\mu E_{i}(z, t)}{\hbar} \\
P_{i, r}(V, U, z, t)= & \epsilon u \gamma \frac{t_{c} \omega_{0}}{\epsilon_{0}} \frac{\mu}{\hbar}\left(\frac{\gamma_{a}{ }^{\prime}-\gamma_{a b}+\gamma_{b}{ }^{\prime}}{2 \gamma \gamma_{a}{ }^{\prime} \gamma_{b}{ }^{\prime}}\right)^{1 / 2} \\
& \times P_{i, r}\left(v, \omega_{\alpha}, z, t\right),
\end{aligned}
$$




$$
\begin{aligned}
& D(V, U, z, t)=\frac{\epsilon u t_{c} \omega_{0}}{\epsilon_{0}} \frac{\mu^{2}}{\hbar} D\left(v, \omega_{\alpha}, z, t\right), \\
& M(V, U, z, t)=\frac{\epsilon u t_{c} \omega_{0}}{\epsilon_{0}} \frac{\mu^{2}}{\hbar} M\left(v, \omega_{\alpha}, z, t\right), \\
& \lambda_{a}(V, U, z, t)=\frac{\epsilon u t_{c} \omega_{0}}{\epsilon_{0}} \frac{\mu^{2}}{\hbar} \lambda_{a}\left(v, \omega_{\alpha}, z, t\right), \\
& \lambda_{b}(V, U, z, t)=\frac{\epsilon u t_{c} \omega_{0}}{\epsilon_{0}} \frac{\mu^{2}}{\hbar} \lambda_{b}\left(v, \omega_{\alpha}, z, t\right),
\end{aligned}
$$

where $\gamma_{a}^{\prime}=\gamma_{a}+\Gamma_{a}$ and $\gamma_{b}{ }^{\prime}=\gamma_{b}+\Gamma_{b}$. New variables $V, U$, $y, y_{0}$, and $\delta$ and are defined as $V=v / \epsilon u, U=\left(\omega_{\alpha}-\omega_{0}\right) / \gamma, y=$ $\left(\omega-\omega_{0}\right) / \gamma, y_{0}=\left(\Omega-\omega_{0}\right) / \gamma$, and $\delta=\gamma / \gamma_{c}$, respectively.

The resulting equations using the new variables are

$$
\begin{aligned}
\frac{\partial A_{r}(z, t)}{\partial t}+v_{f} \frac{\partial A_{r}(z, t)}{\partial z}= & -\frac{1}{2 t_{c}}\left[A_{r}(z, t)+\delta\left(y-y_{0}\right) A_{i}(z, t)\right. \\
& \left.+\int_{-\infty}^{\infty} \int_{-\infty}^{\infty} P_{i}(V, U, z, t) \mathrm{d} V \mathrm{~d} U\right], \\
\frac{\partial A_{i}(z, t)}{\partial t}+v_{f} \frac{\partial A_{i}(z, t)}{\partial z}= & -\frac{1}{2 t_{c}}\left[A_{i}(z, t)-\delta\left(y-y_{0}\right) A_{r}(z, t)\right. \\
& \left.-\int_{-\infty}^{\infty} \int_{-\infty}^{\infty} P_{r}(V, U, z, t) \mathrm{d} V \mathrm{~d} U\right],
\end{aligned}
$$

$$
\begin{aligned}
& \frac{\partial D(V, U, z, t)}{\partial t}+\epsilon u V \frac{\partial D(V, U, z, t)}{\partial z} \\
& =\lambda_{a}-\lambda_{b}-\frac{\gamma_{a}{ }^{\prime}+\gamma_{a b}+\gamma_{b}^{\prime}}{2} D(V, U, z, t) \\
& \quad-\frac{\gamma_{a}^{\prime}+\gamma_{a b}-\gamma_{b}^{\prime}}{2} M(V, U, z, t)+\frac{2 \gamma_{a}^{\prime} \gamma_{b}^{\prime}}{\gamma_{a}^{\prime}-\gamma_{a b}+\gamma_{b}^{\prime}} \\
& \quad \times\left[A_{r}(z, t) P_{i}(V, U, z, t)-A_{i}(z, t) P_{r}(V, U, z, t)\right] \\
& \quad+\frac{\epsilon \Gamma_{a}}{2 \sqrt{\pi}} \exp \left(-\epsilon^{2} V^{2}\right) \int_{-\infty}^{\infty}\left[M\left(V^{\prime}, U, z, t\right)+D\left(V^{\prime}, U, z, t\right)\right] \mathrm{d} V^{\prime} \\
& \quad-\frac{\epsilon \Gamma_{b}}{2 \sqrt{\pi}} \exp \left(-\epsilon^{2} V^{2}\right) \int_{-\infty}^{\infty}\left[M\left(V^{\prime}, U, z, t\right)-D\left(V^{\prime}, U, z, t\right)\right] \mathrm{d} V^{\prime},
\end{aligned}
$$

$$
\begin{aligned}
& \frac{\partial M(V, U, z, t)}{\partial t}+\epsilon u V \frac{\partial M(V, U, z, t)}{\partial z} \\
& =\lambda_{a}+\lambda_{b}-\frac{\gamma_{a}^{\prime}-\gamma_{a b}-\gamma_{b}^{\prime}}{2} D(V, U, z, t) \\
& \quad-\frac{\gamma_{a}^{\prime}-\gamma_{a b}+\gamma_{b}^{\prime}}{2} M(V, U, z, t)+\frac{\epsilon \Gamma_{a}}{2 \sqrt{\pi}} \exp \left(-\epsilon^{2} V^{2}\right) \\
& \quad \times \int_{-\infty}^{\infty}\left[M\left(V^{\prime}, U, z, t\right)+D\left(V^{\prime}, U, z, t\right)\right] \mathrm{d} V^{\prime}+\frac{\epsilon \Gamma_{b}}{2 \sqrt{\pi}} \\
& \quad \times \exp \left(-\epsilon^{2} V^{2}\right) \int_{-\infty}^{\infty}\left[M\left(V^{\prime}, U, z, t\right)-D\left(V^{\prime}, U, z, t\right)\right] \mathrm{d} V^{\prime},
\end{aligned}
$$

$$
\begin{aligned}
& \frac{\partial P_{r}(V, U, z, t)}{\partial t}+\epsilon u V \frac{\partial P_{r}(V, U, z, t)}{\partial z}=-\gamma\left[P_{r}(V, U, z, t)\right. \\
& \left.+(y-U-V) P_{i}(V, U, z, t)-A_{i}(z, t) D(V, U, z, t)\right], \quad(28) \\
& \quad \frac{\partial P_{i}(V, U, z, t)}{\partial t}+\epsilon u V \frac{\partial P_{i}(V, U, z, t)}{\partial z}=-\gamma\left[P_{i}(V, U, z, t)\right. \\
& \left.-(y-U-V) P_{r}(V, U, z, t)+A_{r}(z, t) D(V, U, z, t)\right] . \quad \text { (29) }
\end{aligned}
$$

Because of the retention of the spatial dependence of the field and polarization amplitudes, the dependent variables are governed by partial differential equations. This is in contrast to the total derivatives used for the study of laser instabilities, ${ }^{55}$ where the boundary condition for a traveling wave with uniform loss is applied. Since self-locking properties of the system are of interest here, a transformation to a reference frame that travels with the self-locked pulses is applied to Eqs. (24)-(29). The following changes of variables are done:

$$
\begin{gathered}
\tau=t-z / v_{s} \\
\eta=z
\end{gathered}
$$

To capture the essence of this approach to self-locking with minimum mathematical complexity, a single-isotope medium and cavity tuning to atomic resonance are assumed. For gain and loss that are uniformly distributed and assuming stationary pulsations so that $\mathrm{d} A_{r} / \mathrm{d} \eta=\mathrm{d} A_{i} / \mathrm{d} \eta=0$, the resulting equations in this traveling-wave reference are

$$
\begin{aligned}
\frac{\mathrm{d} A_{r}(\tau)}{\mathrm{d} \tau}= & -\frac{1}{2 t_{c}\left(1-v_{f} / v_{s}\right)}\left[A_{i}(\tau)+\int_{-\infty}^{\infty} P_{r}(V, \tau) \mathrm{d} V\right], \quad(32) \\
\frac{\mathrm{d} A_{i}(\tau)}{\mathrm{d} \tau}= & -\frac{1}{2 t_{c}\left(1-v_{f} / v_{s}\right)}\left[A_{r}(\tau)-\int_{-\infty}^{\infty} P_{i}(V, \tau) \mathrm{d} V\right], \quad(33) \\
\frac{\partial D(V, \tau)}{\partial \tau}= & \lambda_{a}-\lambda_{b}-\frac{\gamma_{a}{ }^{\prime}+\gamma_{a b}+\gamma_{b}{ }^{\prime}}{2} D(V, \tau) \\
& -\frac{\gamma_{a}{ }^{\prime}+\gamma_{a b}-\gamma_{b}{ }^{\prime}}{2} M(V, \tau)+\frac{2 \gamma_{a}{ }^{\prime} \gamma_{b}{ }^{\prime}}{\gamma_{a}{ }^{\prime}-\gamma_{a b}+\gamma_{b}{ }^{\prime}} \\
& \times\left[A_{r}(\tau) P_{i}(V, \tau)-A_{i}(\tau) P_{r}(V, \tau)\right] \\
& +\frac{\epsilon \Gamma_{a}}{2 \sqrt{\pi}} \exp \left(-\epsilon^{2} V^{2}\right) \int_{-\infty}^{\infty}\left[M\left(V^{\prime}, \tau\right)+D\left(V^{\prime}, \tau\right)\right] \mathrm{d} V^{\prime} \\
& -\frac{\epsilon \Gamma_{b}}{2 \sqrt{\pi}} \exp \left(-\epsilon^{2} V^{2} \int_{-\infty}^{\infty}\left[M\left(V^{\prime}, \tau\right)-D\left(V^{\prime}, \tau\right)\right] \mathrm{d} V^{\prime},\right.
\end{aligned}
$$

$$
\begin{aligned}
\frac{\partial M(V, \tau)}{\partial \tau}= & \lambda_{a}+\lambda_{b}-\frac{\gamma_{a}{ }^{\prime}-\gamma_{a b}-\gamma_{b}{ }^{\prime}}{2} D(V, \tau) \\
& -\frac{\gamma_{a}{ }^{\prime}-\gamma_{a b}+\gamma_{b}{ }^{\prime}}{2} M(V, \tau)+\frac{\epsilon \Gamma_{a}}{2 \sqrt{\pi}} \exp \left(-\epsilon^{2} V^{2}\right) \\
& \times \int_{-\infty}^{\infty}\left[M\left(V^{\prime}, \tau\right)+D\left(V^{\prime}, \tau\right)\right] \mathrm{d} V^{\prime}+\frac{\epsilon \Gamma_{b}}{2 \sqrt{\pi}} \\
& \times \exp \left(-\epsilon^{2} V^{2} \int_{-\infty}^{\infty} M\left(V^{\prime}, \tau\right)-D\left(V^{\prime}, \tau\right) \mathrm{d} V^{\prime},\right.
\end{aligned}
$$


$\frac{\mathrm{d} P_{r}(V, \tau)}{\mathrm{d} \tau}=-\gamma\left[P_{r}(V, \tau)+(y-V) P_{r}(V, \tau)-A_{i}(\tau) D(V, \tau)\right]$,

$\frac{\mathrm{d} P_{i}(V, \tau)}{\mathrm{d} \tau}=-\gamma\left[P_{i}(V, \tau)-(y-V) P_{i}(V, \tau)+A_{r}(\tau) D(V, \tau)\right]$.

Since the atomic velocities are small compared with the velocity of light, $1+\epsilon u V / v_{s} \approx 1$ has been assumed in Eqs. (34)-(37).

Equations (32)-(37) have been numerically integrated using the Runge-Kutta method and using the same $\gamma, \gamma_{a}, \gamma_{b}$, $\gamma_{a b}, \Gamma_{a}$, and $\Gamma_{b}$ and $\lambda_{b} / \lambda_{a}$ as in Ref. 55. The natural broadening and the Doppler linewidth used are 4.6 and $110 \mathrm{MHz}$, respectively. The $v_{f} / v_{s}$ ratio has been adjusted to give the correct round-trip time corresponding to a cavity length. Since no detectable change in the round-trip time as a function of the excitation current for a fixed cavity length has been observed, $v_{f} / v_{s}$ has also been adjusted to reflect this.

Typical intensity plots of the signal for $r=1.25$ and $r=2.0$ are shown in Fig. 10. The cavity decay rate, $1 / 2 t_{c}$, used is $0.02 \times 10^{9}$. Both plots show the ringing feature that has been observed experimentally. For $r=1.25$ the pulsations
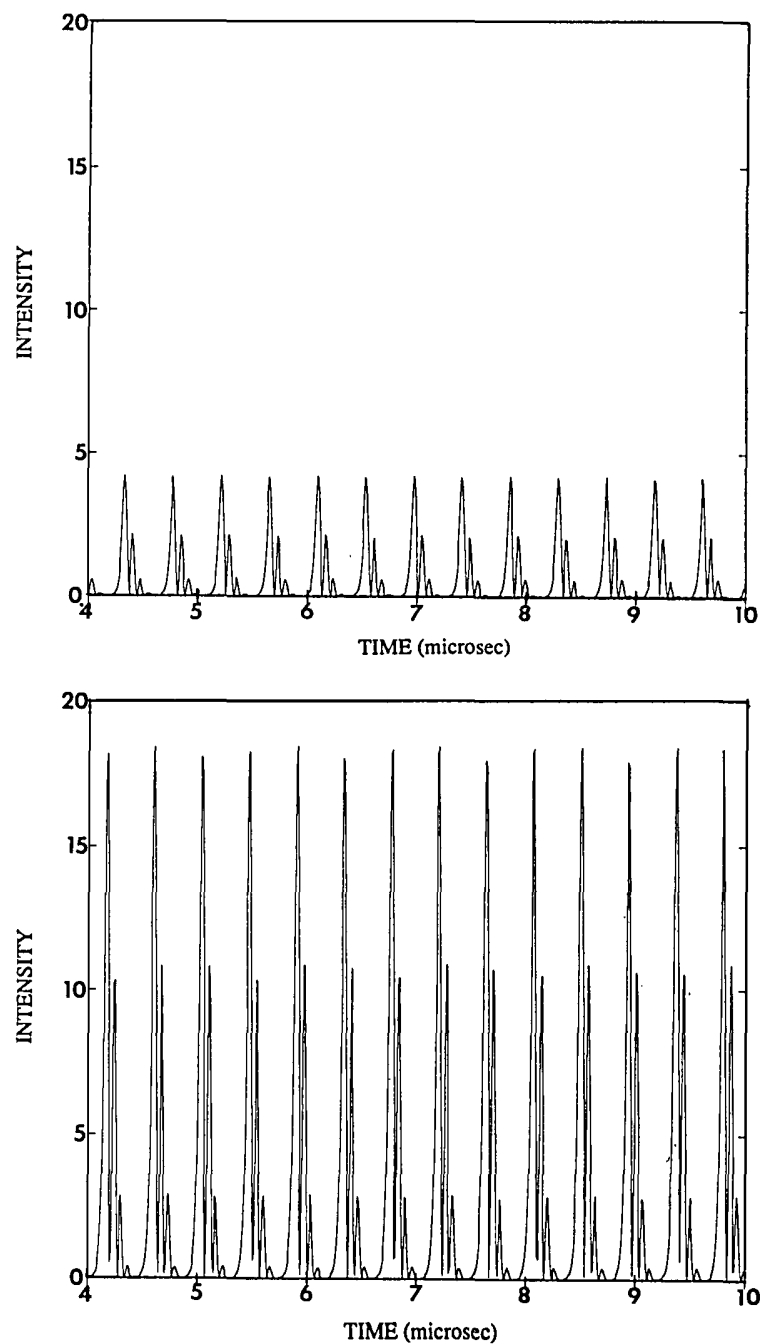

Fig. 10. Numerically generated plot of the intensity of the signal for (a) $r=1.25$ and (b) $r=2.0$. are almost periodic, which is consistent with the experimental observation of periodic pulsations close to threshold. (See Figs. 5 and 7.) Although the numerical solutions predict modulation of the pulses for higher currents, they do not reproduce the rich dynamical features observed experimentally, such as the changing number of pulses in a cavity round-trip time. This shortcoming of the solutions may be attributed to the stringent assumption of stationary modelocked pulsations.

\section{CONCLUSION}

Our experiments on the self-locking in long-cavity xenon lasers have been described. Our results show that the number of pulses in a round-trip time increases with increasing excitation current and cavity length. Other significant dynamical effects that have been observed include the ringing and the modulation of the pulses. A semiclassical model for self-locking in inhomogeneously broadened systems has also been presented. Our numerical results based on this model agree with the experimental observations of the pulse ringing and periodic pulsations close to threshold. Our future theoretical work on self-locking will focus on the improvement of our semiclassical model to yield agreement with the full range of experimental data reported here.

\section{ACKNOWLEDGMENT}

This research was supported in part by the National Science Foundation under grant ECS-8511593 and by Tektronix, Inc.

* Permanent address, National Institute of Physics, University of the Philippines, Quezon City, The Philippines.

\section{REFERENCES}

1. P. W. Smith, Proc. IEEE 58, 1342 (1970).

2. L. Allen and D. G. C. Jones, in Progress in Optics, E. Wolf, ed. (North-Holland, Amsterdam, 1971), Vol. IX, pp. 181-234.

3. P. W. Smith, Appl. Phys. Lett. 10, 51 (1967).

4. A. Tanaka, A. Mori, Y. Homma, M. Yamanaka, and H. Yoshinaga, Jpn. J. Appl. Phys. 12, 1650 (1973).

5. M. H. Crowell, IEEE J. Quantum Electron. QE-1, 1 (1965).

6. M. H. McClure, Appl. Phys. Lett. 7, 148 (1965).

7. O. L. Gaddy and E. M. Schaeffer, Appl. Phys. Lett. 9, 281 (1966).

8. P. W. Smith, IEEE J. Quantum Electron. QE-11, 627 (1967).

9. P. W. Smith, Appl. Phys. Lett. 13, 235 (1968).

10. H. Statz, G. A. DeMars, and C. L. Tang, J. Appl. Phys. 38, 2212 (1967).

11. F. R. Nash, IEEE J. Quantum Electron. QE-3, 189 (1967).

12. T. Uchida and A. Ueki, IEEE J. Quantum Electron. QE-3, 17 (1967).

13. T. Uchida and T. E. Rozzi, IEEE J. Quantum Eletron. QE-3, 7 (1967).

14. K. Kohiyama, T. Fujioka, and M. Kobayashi, Proc. IEEE 56, 333 (1968).

15. T. A. Bridges and P. K. Cheo, Phys. Lett. 14, 262 (1969).

16. G. R. Willenbring and J. A. Carruthers, J. Appl. Phys. 41, 5040 (1970).

17. H. H. Kim and H. Marantz, IEEE J. Quantum Electron. QE-6, 749 (1970).

18. A. Pardue, G. Dezenberg, and O. P. McDuff, IEEE J. Quantum Electron. QE-7, 22 (1971).

19. L. Casperson, Ph.D. dissertation (California Institute of Technology, Pasadena, Calif., 1971).

20. A. V. Mochalov and D. K. Mynbaev, Sov. Phys. Tech. Phys. 18, 422 (1973). 
21. A. Haug and J. R. Whinnery, IEEE J. Quantum Electron. QE$10,406(1974)$.

22. C. N. Y. Chow, G. R. Carruthers, and G. R. Willenbring, J. Appl. Phys. 48, 1976 (1977).

23. C. Halford and C. W. Bray, Opt. Quantum Electron. 12, 309 (1980).

24. C. O. Weiss and H. King, Opt. Commun. 44, 59 (1982).

25. C. O. Weiss, A. Godone, and A. Olafsson, Phys. Rev. A 28, 892 (1983).

26. N. J. Halas, S.-N. Liu, and N. B. Abraham, Phys. Rev. A 28, 2915 (1983).

27. Y. I. Chetverikov, Sov. Tech. Phys. Lett. 11, 190 (1985).

28. M. A. Duguay, S. L. Shapiro, and P. M. Rentzepis, Phys. Rev. Lett. 19, 1014 (1967)

29. S. L. Shapiro, M. A. Duguay, and L. B. Kreuzer, Appl. Phys. Lett. 12, 36 (1968).

30. M. Bass and D. Woodward, Appl. Phys. Lett. 12, 275 (1968).

31. K. Otsuka, IEEE J. Quantum Electron. QE-14, 639 (1978).

32. L. A. Lugiato, C. Oldano, and L. M. Narducci, J. Opt. Soc. Am. B 5,879 (1988).

33. M. D. Sayers and L. Allen, Phys. Rev. A 1, 1730 (1970).

34. S. A. Gonchukov, V. Ermachenko, and E. D. Protsenko, Zh. Eksp. Teor. Fiz. (USSR) 65, 487 (1973) [Sov. Phys. JETP 28, 239 (1974)].

35. W. Lamb, Phys. Rev. 134, A1429 (1964).

36. A. G. Fox and P. W. Smith, Phys. Rev. Lett. 18, 826 (1967).

37. H. Statz and C. L. Tang, J. Appl. Phys. 36, 3923 (1965).

38. H. Statz, G. A. DeMars, and C. L. Tang, J. Appl. Phys. 38, 2212 (1967).
39. C. L. Tang and H. Statz, J. Appl. Phys. 39, 31 (1968); 40, 377 (1969).

40. A. Bambini and P. Burlamacchi, IEEE J. Quantum Electron. QE-4, 101 (1968).

41. H. Risken and K. Nummedal, J. Appl. Phys. 39, 4662 (1963).

42. R. Graham, Phys. Lett. 58A, 449 (1976).

43. M. Piche, Phys. Can. 40, 31 (1984).

44. W. Brunner, R. Fischer, and H. Paul, J. Opt. Soc. Am. B 2, 202 (1985).

45. N. A. Kotomtseva, N. A. Loiko, and A. M. Samson, J. Opt. Soc. Am. B 2, 232 (1985).

46. N. B. Abraham, L. A. Lugiato, and L. M. Narducci, eds., Special issue on instabilities in active optical media, J. Opt. Soc. Am. B 2(1) (1985).

47. F. T. Arecchi and R. G. Harrison, eds., Instabilities and Chaos in Quantum Optics (Springer-Verlag, Berlin, 1987).

48. R. W. Boyd, M. G. Raymer, and L. M. Narducci, eds., Optical Instabilities (Cambridge U. Press, Cambridge, 1986).

49. T. K. Lim and B. K. Garside, IEEE J. Quantum Electron QE-8, 45 (1972).

50. C. E. Halford, J. Appl. Phys. 44, 5644 (1973).

51. T. Miyashita and J. Ikenuoe, Jpn. J. Appl. Phys. 15, 99 (1976).

52. P. S. Landa and V. A. Vygodin, Sov. J. Quantum Electron. 7, 428 (1977).

53. G. J. Linford, E. R. Peressini, W. R. Sooy, and M. L. Spaeth, Appl. Opt. 13, 379 (1974).

54. L. W. Casperson, IEEE J. Quantum Electron. QE-14, 756 (1978).

55. L. W. Casperson, J. Opt. Soc. Am. B 2, 62 (1985). 